\title{
Associação das condições normativas e subjetivas de saúde bucal com a insatisfação com os serviços odontológicos entre adultos brasileiros
}

\author{
The association between normative and subjective oral health \\ conditions and dissatisfaction with dental services \\ among adult Brazilians
}

Diego Figueiredo Nóbrega ${ }^{1}$

João Gabriel Silva Souza ${ }^{1}$

Ana Camila Batista Medeiros de Assis ${ }^{1}$

Andréa Maria Eleutério de Barros Lima Martins ${ }^{2}$

Jaqueline Vilela Bulgareli ${ }^{1}$

\footnotetext{
${ }^{1}$ Faculdade de Odontologia de Piracicaba, Universidade Estadual de Campinas. Av. Limeira 901, Areão. 13414 903 Piracicaba SP Brasil. diego_duke@hotmail.com ${ }^{2}$ Departamento de Odontologia, Centro de Ciências Básicas e da Saúde, Universidade Estadual de Montes Claros. Montes Claros MG Brasil.
}

\begin{abstract}
The prevalence of dissatisfaction with dental services and its association with normative and subjective oral health conditions among adult Brazilians was evaluated. This cross-sectional study used data from the last Brazilian National Oral Health Survey (SBBrasil), conducted in 2010. It was considered a representative sample of the adult Brazilian population. Data were analyzed by descriptive, univariate and multiple statistics (OR / 95\% CI), with correction by the design effect. Among the 4539 adults included in the study, 614 (13.4\%) reported dissatisfaction with dental services. Dissatisfaction was higher among adults who had any decayed teeth (1.61 / 1.06; 2.44), adults who were dissatisfied with their teeth and mouth $(2.36 / 1.39 ; 4.02)$ and adults who had reported toothache in the previous six months $(1.99 / 1.29,3.07)$. The conclusion drawn was that the prevalence of dissatisfaction with dental services among Brazilian adults was low and associated to normative and subjective oral health conditions.
\end{abstract}

Key words Patient satisfaction, Adult, Cross-sectional studies, Dental care, Health evaluation
Resumo Avaliou-se a prevalência da insatisfação com os serviços odontológicos e sua associação com condições normativas e subjetivas de saúde bucal entre adultos brasileiros. Trata-se de um estudo transversal que utilizou dados do inquérito nacional de saúde bucal (SB Brasil) realizado em 2010. Considerou-se uma amostra representativa da população adulta brasileira. Foram conduzidas análises descritivas, bivariadas e múltiplas (OR/ IC 95\%) com correção pelo efeito desenho. Dentre os 4.539 adultos incluídos, 614 (13,4\%) relataram a insatisfação com os serviços odontológicos utilizados. A insatisfação foi maior entre aqueles que apresentavam algum dente cariado (1,61/1,06; 2,44), que estavam insatisfeitos com seus dentes e boca $(2,36 / 1,39 ; 4,02)$ e que haviam relatado dor de dente nos últimos 6 meses $(1,99 / 1,29 ; 3,07)$. Conclui-se que a prevalência da insatisfação com os serviços odontologicos entre adultos brasileiros foi baixa e esteve associada a condições normativas e subjetivas de saúde bucal.

Palavras-chave Satisfação do paciente, Adulto, Estudos transversais, Assistência odontológica, Avaliação em saúde 


\section{Introdução}

O estudo da qualidade da assistência à saúde prestada a população tem sido adotado como uma importante ferramenta para o planejamento, avaliação e organização dos serviços de saúde, tanto em países desenvolvidos ${ }^{1,2}$, como naqueles em desenvolvimento ${ }^{3,4}$. Contudo, quando se avalia a qualidade de um determinado serviço, a opinião do usuário, sem dúvida, é um elemento determinante a ser considerado ${ }^{5}$. Dessa forma, a satisfação do usuário pode ser considerada um dos principais objetivos a ser alcançado pelos serviços de saúde, sendo, portanto, um instrumento de avaliação permanente da qualidade do serviço prestado $^{6}$. A percepção/satisfação do usuário em relação ao serviço de saúde utilizado tem sido foco de estudos prévios, tanto na área da assistência médica ${ }^{2,7-9}$, como odontológica ${ }^{3,4,10,11} \cdot \mathrm{Na}$ odontologia, o desenvolvimento de estudos focados no tema "satisfação do paciente" demonstra a preocupação crescente de uso deste indicador no processo de avaliação da qualidade da assistência ${ }^{3,4,10-14}$, uma vez que a avaliação dos serviços odontológicos sob a perspectiva dos usuários superaria as limitações de avaliações meramente técnicas, permitindo o desenvolvimento de ações mais direcionadas às reais necessidades de saúde da população.

No Brasil, o interesse pelo estudo da satisfação dos usuários com os serviços odontológicos está diretamente ligado à criação e ao fortalecimento do Sistema Único de Saúde (SUS) e à ampliação da assistência odontológica pública no país, que incentiva a participação popular nos processos de planejamento e avaliação das ações de saúde, por meio do controle social ${ }^{15}$. Desde então, a satisfação dos usuários com os serviços odontológicos tem sido avaliada constantemente, por meio de inquéritos epidemiológicos nacionais sobre as condições de saúde bucal da população brasilei$\mathrm{ra}^{16,17}$. No levantamento de 2003, a prevalência da avaliação do serviço odontológico como "bom", ou "muito bom" variou entre $73,6 \%$ e $83,3 \%$, de acordo com as faixas etárias ${ }^{16}$. Já no estudo de 2010, a avaliação positiva do atendimento odontológico, variou de $83,9 \%$ a $87,3 \%$, em função da faixa etária ${ }^{17}$. No entanto, estudos prévios para identificar fatores associados à satisfação/ insatisfação com a assistência odontológica no Brasil têm sido realizados principalmente com o contingente populacional idoso $\mathrm{o}^{3,4}$, salientando a necessidade de avaliação dessa problemática na população adulta brasileira, a qual possui condições de saúde bucal preocupantes ${ }^{17}$.
Ressalta-se que diferentes fatores têm sido associados à satisfação/insatisfação dos usuários, tais como condiçôes sociodemográficas ${ }^{2,4,7,18-20}$, comportamentos ${ }^{3,4,21}$ e desfechos em saúde bucal $^{3,4,21,22}$. Os desfechos em saúde bucal podem ser divididos em condições normativas e subjetivas. As condições normativas são medidas objetivas do estado de saúde bucal avaliadas pelo profissional. Por outro lado, as condições subjetivas dizem respeito a julgamentos pessoais, ou seja, a situação de saúde bucal autopercebida pelos indivíduos $^{23}$. A associação entre satisfação/insatisfação com desfechos em saúde bucal na população brasileira, tem sido encontrada apenas para condições subjetivas de saúde bucal ${ }^{3,421}$. Portanto, não tem sido bem esclarecida na literatura a possibilidade de associação das condições normativas de saúde bucal com a insatisfação do usuário, principalmente ao considerar uma amostra representativa da população brasileira.

Ao considerar os diferentes fatores que podem estar associados à avaliação da qualidade dos serviços de saúde, destaca-se na literatura o modelo teórico proposto por Andersen e David$\operatorname{son}^{24}$, que organiza as variáveis independentes nos subgrupos: ambiente externo, características individuais, comportamentos e desfechos (objetivos e subjetivos) relacionados à saúde. Assim, os determinantes primários da saúde bucal (características do ambiente externo e características individuais da população e do serviço de saúde) influenciariam nos comportamentos. Os comportamentos, por sua vez, teriam influência nos desfechos objetivos e subjetivos de saúde bucal. No entanto, há de se considerar o dinamismo do modelo, uma vez que os determinantes primários e comportamentais além de serem preditores, também podem ser vistos como uma consequência dos desfechos em saúde (fenômeno de retroalimentação $)^{24}$. Ou seja, os desfechos podem ser determinados, mas também determinariam os comportamentos em saúde bucal.

Considera-se que a percepção da população adulta brasileira sobre os serviços odontológicos é de fundamental interesse para a avaliação e planejamento em saúde. Além disso, não têm sido relatado na literatura estudos prévios que tenham relacionado a insatisfação da população adulta brasileira com os serviços odontológicos e os principais desfechos em saúde bucal (condições normativas e subjetivas). Dessa forma, o objetivo do presente trabalho foi estimar a prevalência da insatisfação com os serviços odontológicos em uma amostra representativa dos adultos brasileiros e se esta avaliação está associada 
às condições normativas e subjetivas de saúde bucal. Considerou-se o modelo teórico proposto por Andersen e Davidson ${ }^{24}$.

\section{Metodologia}

Trata-se de um estudo transversal que utilizou dados da Pesquisa Nacional de Saúde Bucal (SBBrasil 2010), disponibilizados publicamente pelo Ministério da Saúde ${ }^{17}$. A pesquisa incluiu exames bucais e entrevistas domiciliares de uma amostra representativa da população brasileira nas faixas etárias índices recomendadas pela $\mathrm{OMS}^{25}$, nas cinco macrorregiões brasileiras. Foram avaliadas as condições de saúde bucal, demográficas e socioeconômicas, uso de serviços odontológicos e questões subjetivas de saúde bucal. No presente estudo foi considerada apenas a amostra representativa da população adulta (3544 anos).

Utilizou-se a amostragem probabilística por conglomerados, em múltiplos estágios, com probabilidade proporcional ao tamanho e considerando um efeito de desenho (deff) igual a 2,0. Cada capital das unidades federativas mais o Distrito Federal $(\mathrm{n}=27)$ e uma amostra de municípios do interior de cada macrorregião brasileira $(\mathrm{n}=5)$ foram consideradas domínios da pesquisa (total $=32$ ). Os municípios e setores censitários foram sorteados pela técnica de Probabilidade Proporcional ao Tamanho ${ }^{26}$.

Os exames e entrevistas foram realizados por cirurgiões-dentistas previamente treinados e calibrados pela técnica do consenso, sendo o valor mínimo aceitável de kappa para cada examinador, grupo etário e agravo estudado igual a $0,65^{26,27}$. As entrevistas foram realizadas com auxílio de computador de mão (Personal Digital Assistant). No total foram examinados e entrevistados, em seus domicílios, 9.564 adultos (de 35 a 44 anos). Neste estudo, foi utilizado um recorte do banco de dados, sendo incluídos aqueles adultos que haviam consultado o dentista há menos de um ano e que responderam a questão relativa a avaliação da consulta odontológica.

A variável dependente - avaliação da consulta odontológica - foi construída a partir da seguinte pergunta "O que o sr(a) achou do tratamento na última consulta? (1-Muito Bom; 2-Bom; 3-Regular; 4-Ruim; 5-Muito Ruim)". Esta variável foi dicotomizada conforme estudos prévios em "insatisfeito" (péssimo ou ruim ou regular) ou "satisfeito" (bom ou ótimo) ${ }^{3,4}$. As demais variáveis independentes foram reunidas em cinco grupos principais, de acordo com o modelo proposto por Andersen e Davidson ${ }^{24}$ : ambiente externo, características individuais, comportamentos e desfechos em saúde, esta última considerando condições normativas e subjetivas de saúde bucal.

As características do ambiente externo estudadas foram: localização geográfica (capital ou interior) e local da consulta (Particular/Plano de saúde/Convênios/Outros ou Serviço público SUS). Com relação às características individuais da população, foram consideradas a idade (3539 ou 40-44 anos - agrupada de acordo com a mediana); raça autodeclarada (Branco ou Negro/ Amarelo/Pardo/Indígena); escolaridade (0-4 ou 5 ou mais anos) e renda familiar (até 500 reais ou mais de 500 reais). No que diz respeito aos comportamentos em saúde, considerou-se a variável motivo da consulta, classificada em "rotina" (Revisão/Prevenção) ou "tratamento" (Dor/ Extração/Tratamento/Outros).

Com relação aos desfechos em saúde, as condições normativas de saúde bucal avaliadas foram: Presença de cárie ("não"- nenhum dente cariado, ou "sim"- 1 ou mais dentes cariados); Perda dentária ("não"- nenhum dente perdido, ou "sim"- 1 ou mais dentes perdidos); e Condição Periodontal ("Não comprometida"- Hígido, ou "Comprometida"- Presença de Sangramento/ Cálculo/Bolsa Periodontal). Já as condições subjetivas de saúde bucal foram: Autopercepção da necessidade de tratamento; Dor de dente nos últimos 6 meses e Satisfação com dentes e boca, classificada em "Satisfeito" (Muito Satisfeito/Satisfeito) ou "Insatisfeito" (Nem satisfeito nem insatisfeito/Insatisfeito/Muito insatisfeito).

$\mathrm{Na}$ análise dos dados, empregou-se o software SPSS ${ }^{\circledR}$ Statistics 18.0. Como o estudo envolvia uma amostragem complexa por conglomerados, utilizou-se a correção pelo efeito de desenho amostral, levando em consideração o efeito dos conglomerados e a atribuição de ponderações desiguais aos elementos amostrados. Para as variáveis categóricas, a análise descritiva incluiu a frequência absoluta $(\mathrm{n})$, a frequência relativa com correção pelo efeito de desenho $\left(\%^{*}\right)$ e erro padrão (EP). Na análise bivariada e na regressão logística, conduzidas para identificar fatores associados à variável dependente, foram feitas estimativas com a correção pelo efeito do desenho amostral. Foram estimados odds ratio e intervalos de confiança de 95\% (OR/IC 95\%) e o valor "p". O nível de significância adotado foi de $20 \%$ $(\mathrm{p} \leq 0,20)$ nas análises bivaridas (para seleção das variáveis independentes que iriam compor o modelo múltiplo) e de $5 \%(\mathrm{p} \leq 0,05)$ na análise múl- 
tipla/regressão logística. O modelo múltiplo final foi ajustado, mantendo-se somente as variáveis independentes associadas à variável dependente. O levantamento epidemiológico "SB Brasil 2010" foi conduzindo, respeitando os princípios éticos da Resolução do Conselho Nacional de Saúde $n^{\circ} 196 / 96$, sendo aprovado e registrado pela Comissão Nacional de Ética em Pesquisa (Conep).

\section{Resultados}

Dos 9.564 adultos examinados no SB Brasil 2010, 4.539 satisfizeram os critérios de inclusão estabelecidos. Destes, 614 (13,4\%) relataram a insatisfação com os serviços odontológicos utilizados. A idade média dos participantes foi de 39,58 anos (EP 0,14), enquanto a escolaridade média foi de 9,51 anos de estudo (EP 0,23). Quanto à distribuição da amostra, a maioria dos adultos era do sexo feminino, tinha entre 40 e 44 anos de idade, se autodeclarou como branco, havia estudado por mais de 5 anos, declarou renda per capita maior do que $\mathrm{R} \$ 500,00$ e residia predominantemente no interior. O sistema de atenção à saúde mais utilizado foi o particular e o principal motivo da procura por atendimento odontológico foi para tratamento (Tabela 1).

$\mathrm{Na}$ análise bivariada (Tabela 2), verificou-se que a avaliação negativa dos serviços odontológicos esteve associada estatisticamente $(\mathrm{p} \leq 0,20)$ a variáveis pertencentes às condições sociodemográficas (escolaridade e renda familiar), comportamentais (motivo do uso), normativas (presença de cárie e doença periodontal) e subjetivas em saúde bucal (autopercepção da necessidade de tratamento; satisfação com dentes e boca e dor de dente).

Já na análise múltipla (Tabela 3), observouse que a prevalência de insatisfação com o atendimento odontológico, independentemente das demais variáveis, foi maior entre os adultos que apresentavam algum dente cariado, que relataram insatisfação com seus dentes e boca e que haviam relatado dor de dente nos últimos 6 meses $(\mathrm{p} \leq 0,05)$.

\section{Discussão}

De uma forma geral, a prevalência da insatisfação com os serviços odontológicos na amostra de adultos brasileiros avaliada foi baixa $(13,4 \%)$. Este resultado está de acordo com aquele encontrado para a população adulta no levantamento nacional de $2003(13,9 \%)^{16}$ e em estudo prévio com indivíduos maiores de 18 anos de idade $(7,9 \%)^{28}$. Resultados semelhantes também foram identificados entre idosos brasileiros, onde a avaliação negativa dos serviços odontológicos variou entre $7,9 \%$ e $14,7 \% 0^{3,4}$. De fato, a baixa prevalência da insatisfação dos usuários com os serviços de saúde em geral é um fenômeno esperado, pois tem sido relatado tanto no Brasil ${ }^{20,29-31}$, quanto na literatura internacional ${ }^{2,7,8,32}$. Alguns autores classificam esse fenômeno como "elevação" das taxas de satisfação, sendo observado mesmo quando as expectativas dos usuários em relação aos serviços são negativas ${ }^{33,34}$. De acordo com estes autores, os pacientes sentem-se satisfeitos independentemente da qualidade do cuidado recebido e a insatisfação só é relatada em eventos extremamente negativos. Além disto, a baixa prevalência de insatisfação pode estar relacionada ao "viés de gratidão", uma vez que os pacientes, seja por medo de perder o acesso, ou pela relação de dependência que têm junto aos profissionais de saúde, evitariam criticar os servi$\operatorname{ços}^{35,36}$. Por outro lado, a baixa prevalência de insatisfação encontrada pode ser um indicativo de que o serviço odontológico prestado é resolutivo e eficiente, além de ser um indicador positivo de qualidade. Entretanto, a caracterização dos insatisfeitos é importante para o aprimoramento da assistência odontológica no país.

Com relação aos fatores que influenciaram a avaliação negativa dos serviços odontológicos, a análise múltipla demonstrou que, dos cinco grupos sugeridos por Andersen e Davidson ${ }^{24}$, apenas as variáveis relacionadas aos desfechos em saúde bucal (condições normativas e subjetivas) estiveram associadas.

A insatisfação com o atendimento odontológico foi maior entre os adultos que apresentavam algum dente cariado no momento do exame clínico. Teoricamente, tem sido descrito na literatura $^{9,24}$ a possibilidade dos desfechos clínicos de saúde bucal influenciarem na satisfação/ insatisfação dos pacientes em relação ao serviço prestado. No entanto, esta associação não havia sido comprovada anteriormente por estudos epidemiológicos em adultos brasileiros e foi confirmada no presente estudo. Tal associação pode indicar uma não resolutividade do serviço, seja no tratamento clínico das lesões de cárie (ação terapêutica), assim como no incentivo a mudanças de comportamentos (ação preventiva) com intuito de controlar os fatores determinantes da doença (acúmulo de biofilme e exposição à açúcar). Portanto, estes fatores devem ser considera- 
Tabela 1. Distribuição da amostra, segundo as variáveis estudadas em adultos brasileiros de 35 - 44 anos, 2010 (n $=4539$ ).

\begin{tabular}{|c|c|c|c|}
\hline \multirow{2}{*}{$\begin{array}{c}\text { Variável } \\
\text { Variável Dependente }\end{array}$} & \multicolumn{2}{|c|}{ Distribuição da amostra } & \multirow[b]{2}{*}{$\mathrm{EP}$} \\
\hline & $\mathbf{n}$ & $\%^{*}$ & \\
\hline \multicolumn{4}{|l|}{ Avaliação do serviço odontológico } \\
\hline Satisfeito & 3925 & 86,6 & \\
\hline Insatisfeito & 614 & 13,4 & 1,2 \\
\hline \multicolumn{4}{|l|}{ Ambiente Externo } \\
\hline \multicolumn{4}{|l|}{ Localização geográfica } \\
\hline Capital & 3510 & 33,8 & \\
\hline Interior & 1029 & 66,2 & 3,7 \\
\hline \multicolumn{4}{|l|}{ Local da consulta ${ }^{\text {a }}$} \\
\hline Particular & 2974 & 67,8 & \\
\hline SUS & 1563 & 32,2 & 2,7 \\
\hline \multicolumn{4}{|l|}{ Características Individuais } \\
\hline \multicolumn{4}{|l|}{ Idade } \\
\hline $35-39$ & 2408 & 48,9 & \\
\hline $40-44$ & 2131 & 51,1 & 2,3 \\
\hline \multicolumn{4}{|l|}{ Sexo } \\
\hline Masculino & 1398 & 34,0 & \\
\hline Feminino & 3141 & 66,0 & 1,8 \\
\hline \multicolumn{4}{|l|}{ Raça autodeclarada } \\
\hline Branco & 2122 & 52,8 & \\
\hline Negro/Amarelo/Pardo/Indígena & 2417 & 47,2 & 2,5 \\
\hline \multicolumn{4}{|l|}{ Escolaridade ${ }^{a}$} \\
\hline $0-4$ anos & 574 & 15,0 & \\
\hline 5 ou mais & 3945 & 85,0 & 1,8 \\
\hline \multicolumn{4}{|l|}{ Renda familiar ${ }^{a}$} \\
\hline Mais de 500 reais & 3927 & 88,2 & \\
\hline Até 500 reais & 511 & 11,8 & 1,1 \\
\hline \multicolumn{4}{|l|}{ Comportamentos } \\
\hline \multicolumn{4}{|l|}{ Motivo do uso a } \\
\hline Rotina & 1289 & 28,0 & \\
\hline Tratamento & 3249 & 72,0 & 1,7 \\
\hline \multicolumn{4}{|l|}{ Desfechos de Saúde } \\
\hline \multicolumn{4}{|l|}{ Condições Normativas } \\
\hline \multicolumn{4}{|l|}{ Dentes com cárie ${ }^{a}$} \\
\hline Não & 2536 & 61,4 & \\
\hline Sim & 1906 & 38,6 & 1,9 \\
\hline \multicolumn{4}{|l|}{ Dentes perdidos ${ }^{a}$} \\
\hline Não & 734 & 22,1 & \\
\hline Sim & 3708 & 77,9 & 2,0 \\
\hline \multicolumn{4}{|l|}{ Condição periodontal a } \\
\hline Não comprometida & 984 & 31,4 & \\
\hline Comprometida & 2136 & 68,6 & 2,3 \\
\hline \multicolumn{4}{|l|}{ Condições subjetivas } \\
\hline Autopercepção da necessidade de tr & & & \\
\hline Não & 1180 & 27,8 & \\
\hline Sim & 3298 & 72,2 & 1,9 \\
\hline Satisfação com dentes e boca ${ }^{a}$ & & & \\
\hline Satisfeito & 2092 & 47,7 & \\
\hline Insatisfeito & 2433 & 52,3 & 2,3 \\
\hline Dor de dente nos últimos 6 meses ${ }^{a}$ & & & \\
\hline Não & 3353 & 69,5 & \\
\hline Sim & 1178 & 30,5 & 2,1 \\
\hline
\end{tabular}


Tabela 2. Análise bivariada dos fatores associados à insatisfação com o serviço odontológico em adultos brasileiros de 35 - 44 anos, 2010.

\begin{tabular}{|c|c|c|c|}
\hline \multirow{2}{*}{$\begin{array}{ll} & \text { Variável } \\
\text { Ambiente Externo } & \end{array}$} & \multicolumn{2}{|c|}{ Distribuição da amostra } & \multirow[b]{2}{*}{ p } \\
\hline & Insatisfeito \% & OR (IC95\%) & \\
\hline \multicolumn{4}{|l|}{ Localização geográfica } \\
\hline Capital & 14,2 & 1,00 & \\
\hline Interior & 13,0 & $0,90(0,64-1,28)$ & 0,558 \\
\hline \multicolumn{4}{|l|}{ Local da consulta } \\
\hline Particular & 12,5 & 1,00 & \\
\hline SUS & 15,2 & $1,25(0,87-1,80)$ & 0,233 \\
\hline \multicolumn{4}{|l|}{ Características Indiviuais } \\
\hline \multicolumn{4}{|l|}{ Idade } \\
\hline $35-39$ & 13,9 & 1,00 & \\
\hline $40-44$ & 12,9 & $0,92(0,62-1,37)$ & 0,694 \\
\hline \multicolumn{4}{|l|}{ Sexo } \\
\hline Masculino & 15,3 & 1,00 & \\
\hline Feminino & 12,4 & $1,28(0,80-2,03)$ & 0,297 \\
\hline \multicolumn{4}{|l|}{ Raça autodeclarada } \\
\hline Branco & 12,5 & 1,00 & \\
\hline Amarelo/Pardo/Negro/Indígena & 14,4 & $1,18(0,85-1,64)$ & 0,330 \\
\hline \multicolumn{4}{|l|}{ Escolaridade } \\
\hline $0-4$ anos & 17,3 & 1,00 & \\
\hline 5 ou mais & 12,8 & $0,70(0,42-1,17)$ & 0,168 \\
\hline \multicolumn{4}{|l|}{ Renda familiar } \\
\hline Mais de 500 reais & 12,5 & 1,00 & \\
\hline Até 500 reais & 21,1 & $1,88(1,17-3,03)$ & 0,008 \\
\hline \multicolumn{4}{|l|}{ Comportamentos } \\
\hline \multicolumn{4}{|l|}{ Motivo do uso } \\
\hline Rotina & 10,5 & 1,00 & \\
\hline Tratamento & 14,5 & $1,45(0,95-2,22)$ & 0,079 \\
\hline \multicolumn{4}{|l|}{ Desfechos de Saúde } \\
\hline \multicolumn{4}{|l|}{ Condições Normativas } \\
\hline \multicolumn{4}{|l|}{ Dentes com cárie } \\
\hline Não & 10,3 & 1,00 & \\
\hline Sim & 18,7 & $2,02(1,33-3,07)$ & 0,001 \\
\hline \multicolumn{4}{|l|}{ Dentes perdidos } \\
\hline Não & 11,6 & 1,00 & \\
\hline Sim & 14,1 & $1,25(0,74-2,11)$ & 0,410 \\
\hline \multicolumn{4}{|l|}{ Condição periodontal } \\
\hline Não comprometida & 9,6 & 1,00 & \\
\hline Comprometida & 13,9 & $1,51(0,90-2,55)$ & 0,118 \\
\hline \multicolumn{4}{|l|}{ Condições Subjetivas } \\
\hline \multicolumn{4}{|c|}{ Autopercepção da necessidade de tratamento } \\
\hline Não & 6,1 & 1,00 & \\
\hline Sim & 16,0 & $2,92(1,69-5,03)$ & $<0,001$ \\
\hline \multicolumn{4}{|l|}{ Satisfação com dentes e boca } \\
\hline Satisfeito & 7,2 & 1,00 & \\
\hline Insatisfeito & 19,2 & $3,04(1,84-5,03)$ & $<0,001$ \\
\hline \multicolumn{4}{|l|}{ Dor de dente nos últimos 6 meses } \\
\hline Não & 9,6 & 1,00 & \\
\hline Sim & 21,9 & $2,63(1,76-3,92)$ & $<0,001$ \\
\hline
\end{tabular}


Tabela 3. Análise múltipla dos fatores associados à insatisfação com o serviço odontológico em adultos brasileiros de 35 - 44 anos, 2010.

\begin{tabular}{lcc}
\hline \multicolumn{1}{c}{ Variável } & \multicolumn{2}{c}{$\begin{array}{c}\text { Distribuição da } \\
\text { amostra }\end{array}$} \\
\hline Desfechos de Saúde & OR (IC95\%) & $\mathbf{p}$ \\
\cline { 2 - 3 } Condições Normativas & & \\
Dentes com cárie & 1,00 & \\
$\quad$ Não & $1,61(1,06-2,44)$ & 0,026 \\
$\quad$ Sim & & \\
Condições Subjetivas & & \\
Satisfação com dentes \\
e boca \\
$\quad$ Satisfeito \\
$\quad$ Insatisfeito & 1,00 & \\
Dor de dente nos & $2,36(1,39-4,02)$ & 0,002 \\
últimos 6 meses & & \\
Não & 1,00 & \\
Sim & $1,99(1,29-3,07)$ & 0,002 \\
\hline
\end{tabular}

dos no atendimento do paciente com o intuito de resolver o problema identificado e a consequente melhoria do serviço prestado. Ressalta-se que, as condições normativas de saúde bucal, tais como número de dentes cariados tem influência negativa na autopercepção da saúde bucal ${ }^{37-39}$, sendo o comprometimento desta associado com a insatisfação com os serviços odontológicos ${ }^{4,21}$.

Sabe-se que as condições subjetivas relacionadas à saúde bucal também podem influenciar o desfecho da avaliação dos serviços de saúde ${ }^{24}$. Das 3 condições avaliadas no presente estudo, duas foram associadas à insatisfação com o atendimento odontológico. Observou-se maior insatisfação com os serviços odontológicos entre aqueles adultos que haviam relatado insatisfação com seus dentes e boca. Este resultado corrobora com os achados de estudos anteriores, que também encontraram associação entre a satisfação/ insatisfação com os serviços odontológicos e a autopercepção de saúde bucal ${ }^{3,4,28}$, demonstrando a importância que a percepção do indivíduo sobre a sua própria saúde exerce no momento de avaliar a qualidade dos serviços prestados. Além disso, considera-se que esta autopercepção reflete, em parte, as condições normativas de saúde bucal desses indivíduos, podendo ser esta última consequência da qualidade da assistência prestada. Assim, é possível que a experiência prévia de cárie influencie na insatisfação dos usuários com seus dentes e boca e vice-versa.
Outra condição subjetiva de saúde bucal que esteve diretamente associada com a avaliação negativa dos serviços odontológicos foi a presença de dor de dente nos últimos 6 meses. Este resultado está em concordância com os achados de outros trabalhos, que encontraram associação entre o relato de dor de dente por parte dos usuários e a satisfação com o atendimento odontológico ${ }^{4}$, ou com a autopercepcão da saúde bucal ${ }^{38,40}$. Este dado é preocupante, pois se levarmos em consideração que apenas $28 \%$ dos adultos procuraram o atendimento odontológico para procedimentos de rotina, fica evidente que a busca pelos serviços odontológicos para tratamento de urgência é comum ${ }^{41}$, e que consequentemente este é um dos fatores que geram insatisfação. Além disto, quando a procura pelo atendimento se dá por dor, pouca interação é esperada entre paciente e cirurgião-dentista quanto à educação, instrução e controle de placa bacteriana ${ }^{42}$. Diante disto, sugere-se que os serviços de saúde bucal procurem conduzir abordagens educativas e preventivas após o alívio da sensação dolorosa, possibilitando assim uma maior interação entre pacientes e profissionais, para que esses pacientes possam se apropriar de conhecimentos e hábitos de vida mais saudáveis.

No que diz respeito às limitações deste estudo, deve-se considerar que se trata de uma pesquisa transversal e, portanto, apresenta as limitações inerentes a este tipo de estudo. Neste sentido, não foi possível avaliar o dinamismo das associações entre insatisfação e variáveis independentes (fenômeno de retroalimentação), pois as causas e efeitos mudam ao longo do tempo e, por se tratar de um estudo transversal, a direção dessas associações não pode ser determinada. Existe ainda a limitação relacionada à construção da variável dependente, pois a satisfação dos entrevistados em relação ao serviço odontológico foi definida a partir de uma única pergunta. No entanto, ressalta-se que os resultados aqui apresentados são relevantes, pois foram obtidos em uma amostra probabilística por conglomerados representativa da população adulta brasileira. Além disto, utilizou-se a correção pelo efeito de desenho na análise dos dados, procedimento extremamente recomendado em estudos com amostras complexas ${ }^{43}$.

\section{Conclusão}

Constatou-se baixa prevalência de insatisfação com os serviços odontológicos entre adultos brasileiros. Além disto, identificou-se associação da 
insatisfação com o serviço odontológico e condições normativas (presença de cárie) e subjetivas (insatisfação com dentes e boca e sensibilidade dolorosa) de saúde bucal. A identificação do perfil do usuário insatisfeito pode subsidiar a reformulação de estratégias de saúde que visem a melhoria da qualidade do serviço odontológico prestado.

\section{Colaboradores}

Concepção, delineamento e planejamento do estudo: DF Nóbrega, JGS Souza, JV Bulgareli; Coleta e análise dos dados, DF Nóbrega, JGS Souza, ACBM Assis. Escrita do artigo: DF Nóbrega, JGS Souza, AMEBL Martins, JV Bulgareli. Revisão da versão final do artigo: DF Nóbrega, JGS Souza, ACBM Assis, AMEBL Martins, JV Bulgareli. 


\section{Referências}

1. Otani K, Kurz RS, Harris LE. Managing primary care using patient satisfaction measures. J Healthc Manag 2005; 50(5):311-324.

2. Rahmqvist M, Bara AC. Patient characteristics and quality dimensions related to patient satisfaction. Int $\mathrm{J}$ Qual Health Care 2010; 22(2):86-92.

3. Rodrigues CA, Silva PL, Caldeira AP, Pordeus IA, Ferreira RC, Martins AM. Factors associated with satisfaction with dental services among the elderly. Rev Saude Publica 2012; 46(6):1039-1050.

4. Martins AM, Jardim LA, Souza JG, Rodrigues CA Ferreira RC, Pordeus IA. Is the negative evaluation of dental services among the Brazilian elderly population associated with the type of service? Rev Bras Epidemiol 2014; 17(1):71-90.

5. Serapioni M. Avaliação de qualidade em saúde: a contribuição da sociologia da saúde para superação da polarização entre visão dos usuários e a perspectiva dos profissionais da saúde. Saúde Debate 1999; 23(53):8192.

6. Santos MP. Avaliação da qualidade dos serviços públicos de atenção à saúde da criança sob a ótica do usuário. Rev Bra Enferm 1995; 48(2):109-119.

7. Myburgh NG, Solanki GC, Smith MJ, Lalloo R. Patient satisfaction with health care providers in South Africa: the influences of race and socioeconomic status. Int $\mathrm{J}$ Qual Health Care 2005; 17(6):473-477.

8. Tung YC, Chang GM. Patient satisfaction with and recommendation of a primary care provider: associations of perceived quality and patient education. Int J Qual Health Care 2009; 21(3):206-213.

9. Donabedian A. The quality of care: how can it be assessed? JAMA 1988; 260(12):1743-1748.

10. Peres KG, Peres MA, Boing AF, Bertoldi AD, Bastos JL, Barros AJ. Reduction of social inequalities in utilization of dental care in Brazil from 1998 to 2008. Rev Saude Publica 2012; 46(2):250-258.

11. Butters JN, Willis DO. A comparison of patient satisfaction among current and former dental school patients. J Dent Educ 2000; 64(6):409-415.

12. Leão ATT, Dias K. Avaliação dos serviços de saúde prestados por faculdades de odontologia: a visão do usuário. Rev Bras Odontol Saúde Coletiva 2001; 2(1):40-46.

13. Andrade KLC, Ferreira EF. Avaliação da inserção da odontologia no Programa Saúde da Família de Pompéu (MG): a satisfação do usuário. Cien Saude Colet 2006; 11(1):123-130.

14. Emmi DT, Barroso RFF. Avaliação das ações de saúde bucal no Programa Saúde da Família no distrito de Mosqueiro, Pará. Cien Saude Colet 2008; 13(1):35-41.

15. Crevelim MA, Peduzzi M. Participação da comunidade na equipe de saúde da família: é possível estabelecer um projeto comum entre trabalhadores e usuários? Cien Saude Colet 2005; 10(2):323-331

16. Brasil. Ministério da Saúde (MS). Projeto SB Brasil 2003: condições de saúde bucal da população brasileira 2002-2003: resultados principais. Brasília: MS; 2004.

17. Brasil. Ministério da Saúde (MS). Departamento de Atenção Básica. Sb Brasil 2010. Pesquisa nacional de saúde bucal: Resultados principais. Brasília: MS; 2011.
18. Dolinsky AL, Caputo RK. The role of health care attributes and demographic characteristics in the determination of health care satisfaction. J Health Care Mark 1990; 10(4):31-39.

19. Atkinson S, Haran D. Individual and district scale determinants of users' satisfaction with primary health care in developing countries. Soc Sci Med 2005; 60(3):501-513.

20. Lima-Costa MF, Loyola Filho AI. Fatores associados ao uso e à satisfação com os serviços de saúde entre usuários do Sistema Único de Saúde na Região Metropolitana de Belo Horizonte, estado de Minas Gerais, Brasil. Epidemiol Serv Saúde 2008; 17(4):247-257.

21. Gouveia GC, Souza WV, Luna CF, Souza-Júnior PRB, Szwarcwald CL. Satisfação dos usuários do sistema de saúde brasileiro: fatores associados e diferenças regionais. Rev Bras Epidemiol 2009; 12(3):281-296.

22. Locker D, Gibson B. Discrepancies between self-ratings of and satisfaction with oral health in two older adult populations. Community Dent Oral Epidemiol 2005; 33(4):280-288.

23. Martins AM, Barreto SM, Pordeus IA. Objective and subjective factors related to self-rated oral health among the elderly. Cad Saude Publica 2009; 25(2):421435.

24. Andersen RM, Davidson PL. Ethnicity, aging, and oral health outcomes: a conceptual framework. Adv Dent Res 1997; 11(2):203-209.

25. World Health Organization (WHO). Oral health surveys: basic methods. $4^{\text {th }}$ ed. Geneva: WHO; 1997.

26. Roncalli AG, Silva NN, Nascimento AC, Freitas CHSM, Casotti E, Peres KG, Moura L, Peres MA, Freire MCM, Cortes MIS, Vettore MV, Junior MP, Figueiredo N, Goes PSA, Pinto RS, Marques RAM, Moyses SJ, Reis SCGB, Narvai PC. Aspectos metodológicos do Projeto SBBrasil 2010 de interesse para inquéritos nacionais de saúde. Cad Saude Publica 2012; 28(Supl.):s40-s57.

27. World Health Organization (WHO). Calibration of examiners for oral health epidemiological surveys. Geneva: WHO; 1993.

28. Martins AMEBL, Ferreira RC, Santos Neto PE, Carreiro DL, Souza JGS, Ferreira EF. Insatisfação dos usuários com a assistência odontológica: estudo domiciliar populacional. Rev Saude Publica 2015; 49:51.

29. Santos SAS, Meneghim MC, Pereira AC. Análise da organização da demanda e grau de satisfação do profissional e usuário nas unidades de serviço público odontológico do Município de Campos dos Goytacazes/RJ/ Brasil. Rev Odont UNESP 2007; 36(2):169-174.

30. Lima AC, Cabral ED, Vasconcelos MM. Patient satisfaction at Specialized Dental Clinics in Recife, Pernambuco State, Brazil. Cad Saude Publica 2010; 26(5):9911002.

31. Moimaz SAS, Marques JAM, Saliba O, Garbin CAS, Zina LG, Saliba NA. Satisfação e percepção dos usuários do SUS sobre o serviço Público de Saúde. Physis 2010; 20(4):1419-1440.

32. Fan VS, Burman M, McDonell MB, Fihn SD. Continuity of care and other determinants of patient satisfaction with primary care. J Gen Intern Med 2005; 20(3):226-233. 
33. Williams B, Coyle J, Healy D. The meaning of patient satisfaction: an explanation of high reported levels. Soc Sci Med 1998; 47(9):1351-1359.

34. Esperedião M, Trad LAB. Avaliação da satisfação de usuários: considerações teórico-conceituais. Cad Saude Publica 2006; 22(6):1267-1276.

35. Bernhart MH, Wiadnyana IG, Wihardjo H, Pohan I. Patient satisfaction in developing countries. Soc Sci Med 1999; 48(8):989-996.

36. Vaitsman J, Andrade GRB. Satisfação e responsividade: formas de medir a qualidade e a humanização da assistência à saúde. Cien Saude Colet 2005; 10(3):599-613.

37. Heft MW, Gilbert GH, Shelton BJ, Duncan RP. Relationship of dental status, sociodemographic status, and oral symptoms to perceived need for dental care. Community Dent Oral Epidemiol 2003; 31(5):351-360.

38. Vale EB, Mendes AC, Moreira RS. Self-perceived oral health among adults in Northeastern Brazil. Rev Saude Publica 2013; 47(Supl. 3):98-108.

39. Cavalcanti AL, Rodrigues IS, Melo Silveira IT, Oliveira TB, Almeida Pinto MS, Xavier AF, Castro RD, Padilha WW. Dental caries experience and use of dental services among Brazilian prisoners. Int J Environ Res Public Health 2014; 11(12):12118-12128.

40. Atchison KA, Gift HC. Perceived oral health in a diverse sample. Adv Dent Res 1997; 11(2):272-280.

41. Martins AM, Haikal DS, Pereira SM, Barreto SM. Routine use of dental services by the elderly in Brazil: the SB Brazil Project. Cad Saude Publica 2008; 24(7):16511666.

42. Pinellia C, Turrionib APS, Loffredoa LCM. Autopercepção em higiene bucal de adultos: reprodutibilidade e validade. Rev odontol UNESP 2008; 37(2):163-169.

43. Queiroz RCS, Portela MC, Vasconcellos MTL. Pesquisa sobre as Condições de Saúde Bucal da População Brasileira (SB Brasil 2003): seus dados não produzem estimativas populacionais, mas há possibilidade de correção. Cad Saude Publica 2009; 25(1):47-58.

Artigo apresentado em 12/07/2016

Aprovado em 21/11/2016

Versão final apresentada em 23/11/2016 